\title{
APHIS CRACCIVORA KOCH. AND PREDATORS ON FABA BEAN AND COWPEA IN NEWLY RECLAIMED AREAS IN EGYPT
}

\author{
ALI , SH. A.M. ${ }^{1}$, A.A.A SALEH ${ }^{2}$ and NADIA E. MOHAMED ${ }^{2}$ \\ 1.Piercing \& Sucking Insect Research Dept., Plant Prot. Res. Institute, ARC, Dokki, \\ Giza, Egypt \\ 2. Biological Control Research Dept., Plant Prot. Res. Institute, ARC, Dokki, Giza, Egypt
}

(Manuscript received 12 June 2013)

\begin{abstract}
The population density of the cowpea aphid, Aphis craccivora Koch. and its associated predators on faba bean and cowpea fields were studied at El-Khattara district, Sharkia Governorate, Egypt in 2010-2011 and 2011-2012 seasons. A. craccivora had three peaks on faba bean for each season the first peak was during the fourth week of December, the second one was in the second week of February, where the last peak was in the first week of March (2010-2011 season), The three peaks of 2011-2012 were recorded in the first week of January, the fourth week of February and the second week of March 2012. There was one peak only on cowpea in the third and fourth week of July in 2011 and 2012, respectively. The common predators observed in faba bean and cowpea fields were: Coccinella undecimpunctata L., Chrysoperla carnea Steph., Paederus alfierii (Koch.), Orius sp., Syrphus sp. Scymnus sp. and Cydonia vicina isis (Muls.)

Regarding predators on faba bean, two peaks were found in the first weeks of February and second week of March (37 and 79 predators/ 100 plants) during the first season 2010-2011, also, two peaks were found in the third week of February and second week of March (61 and 58 predators / 100 plants), respectively in 201112 season.

On cowpea, however, also two peaks were found in the fourth week of July and second week of August (89 and 69 predators/ 50 plants), respectively in 2011 season, while, one peak was found in the third week of July (78 predators/ 50 plants) in 2012.

Statistical analysis showed that temperature and relative humidity had different effects (significant or insignificant) on insects under investigation.

Key words: Aphis craccivora, aphid predators, biological control, faba bean , cowpea
\end{abstract}

\section{INTRODUCTION}

Aphids are the most important insect pests of different crops allover the world, (Ibrahim, 1994). On the other hand, faba bean and cowpea plants are of the most important crops in Egypt.

Aphis craccivora Koch., is considered one of the most injurious pests infests these plantations, and other leguminous species causes by sucking the plant sap 
records in Egypt (El-Heneidy et al., 1998, El-Defrawi et al., 2000 , Abdel-Rahman et al., 2005) and in other countries such as Srikanth and Lakkundi, 1990 , Sharma and Yadav, 1994 in India and Kuroli et al., 1999 in Hungary, and indirect damage by transmittion of many virus diseases (El-Defrawi et al., 2000 and Ortiz et al., 2006).

The use of insecticides in controlling aphids generally, leads to many problems, not only increasing resistant strains of aphids to these chemical substances, but also in induction of pollution to man and beneficial insects such as bees and other pollinators, insect parasitoids and predators (Maghraby, 2012).

The relationship between $A$. craccivora infested faba bean and cowpea plants and its aphidophagous insects have drawn the attention of many investigators such as: El-Heneidy et al. (1998), El-Defrawi et al., (2000) and Mandour et al., (2006).

Therefore, this investigation aims to study the role of aphidophagous insects in reducing the cowpea aphid population on faba bean and cowpea plants in the newly reclaimed sandy areas of El-Khattara district, Sharkia Governorate, which may be helpful in (IPM) crops management in faba bean and cowpea crops.

\section{MATERIALS AND METHODS}

\section{The field experiments}

These experiments were carried out to study seasonal abundance of Aphis craccivora Koch., survey and seasonal abundance of insect predators associated with $A$. craccivora and their relative densities, in El-Khattara district, Sharkia Governorate during the two successive growing seasons (faba bean 2010-2011 and 2011-2012, cowpea 2011 and 2012). An area of half feddan was cultivated with faba bean, Vicia faba L., cultivar Giza 2. The sowing dates were in the second week of October in both seasons. In case of cowpea, the cultivated area was also half feddan, was cultivated with cowpea, Vigna unguiculata (L.) cultivar karem 7 were sown in the first week of June 2011 and 2012.

Normal agricultural practices were used in the due time and no chemical control was applied.

\section{The sampling}

One hundred faba bean plants and fifty cowpea plants were weekly examined carefully from seed germination until the end of the season. The samples were examined at random by means of the cross side method, the number of aphid and predators were counted directly in the field and in few cases laboratory rearing was necessary for the predacious immatures till the adults emergence to be identified and counted. 


\section{Climatic factors (temperature and relative humidity):}

Daily records of both maximum and minimum temperatures along with relative humidity were obtained during the periods of the two seasons from the Agrometerological Station at Abou-Kbeer district, which located about 30 k.m. from the experimental farm in El-Khattara district. The relationship between weekly numbers of A. cracciovra, predators and corresponding weekly of temperature and relative humidity, simple correlation values were calculated according to SAS , 1999.

\section{RESULTS AND DISCUSSION}

\section{Population density of the cowpea aphid, Aphis craccivora on faba bean and cowpea plants:}

\subsection{On faba bean plants:}

The data in the first season (2010-2011) showed that the mean population density of $A$. craccivora on faba bean plants gradually increased as the growing season progressed to reach its maximum (31.2 aphids/ plant) in the first week of March, then the mean number of aphids gradually decreased towards the end of the season, (Table, 1).

In the second season (2011/ 2012), the general mean population density of A. craccivora on faba bean plants generally increased during the growing season to reach its peak of 44.07 aphids/ plant in the fourth week of February, (Table, 1).

There were three peaks for each season, they were 8.97, 24.90 and 31.2 individuals /plant during the fourth week of December, the second week of February and the first week of March in the first season, while they were 14.50, 44.07 and 41.2 individuals/ plant during the first week of January, the fourth week of February and the second week of March during the second season. The mean numbers of $A$. craccivora during the two studied seasons were 9.56 and 16.97 individuals/plant respectively Table, (1). The present results are in agreement with the findings of the following El-Defrawi et al., (2000) who mentioned that $A$. craccivora had two main periods of activity with maximum counts 
Table 1. Seasonal abundance of Aphis craccivora on faba bean plants cultivated during the two seasons 2010-2011 and 2011-2012 in the newly reclaimed sandy area, El-Khattara district.

\begin{tabular}{|c|c|c|c|c|c|c|c|}
\hline \multirow{3}{*}{$\begin{array}{c}\text { Date of } \\
\text { inspection } \\
\text { (weeks) }\end{array}$} & \multicolumn{3}{|c|}{$\begin{array}{c}\text { The first season } \\
\text { 2010-2011 }\end{array}$} & \multirow{3}{*}{$\begin{array}{c}\text { Date of } \\
\text { inspection } \\
\text { (weeks) }\end{array}$} & \multirow{2}{*}{\multicolumn{3}{|c|}{$\begin{array}{c}\text { The second season } \\
\text { 2011-2012 } \\
\text { Means }\end{array}$}} \\
\hline & \multicolumn{3}{|c|}{ Means } & & & & \\
\hline & $\begin{array}{l}\text { Aphid / } \\
\text { plant }\end{array}$ & $\begin{array}{c}\text { Temp. } \\
{ }^{\circ} \mathrm{C} \\
\end{array}$ & $\begin{array}{l}\text { R.H. } \\
\%\end{array}$ & & $\begin{array}{l}\text { Aphid } \\
\text { /plant }\end{array}$ & $\begin{array}{c}\text { Temp. } \\
{ }^{\circ} \mathrm{C} \\
\end{array}$ & $\begin{array}{l}\text { R.H. } \\
\%\end{array}$ \\
\hline Nov.,20103 ${ }^{\text {rd }}$ & $1.61+0.5$ & 24.9 & 65.3 & Nov.,20114 ${ }^{\text {th }}$ & $2.30+0.2$ & 21.8 & 61.2 \\
\hline $4^{\text {th }}$ & $1.83+0.7$ & 23.7 & 65.4 & Mean & \multicolumn{3}{|l|}{$2.30+0.2$} \\
\hline Mean & \multicolumn{3}{|l|}{$1.72+0.11$} & Dec. $\quad 1^{\text {st }}$ & $2.91+0.6$ & 22.3 & 66.4 \\
\hline Dec., $\quad 1^{\text {st }}$ & $2.27 \pm 1.2$ & 22.1 & 70.8 & $2^{\text {nd }}$ & $3.45+0.4$ & 19.8 & 61.6 \\
\hline $2^{\text {nd }}$ & $2.90+0.9$ & 21.7 & 61.6 & $3^{\text {rd }}$ & $3.86 \pm 1.0$ & 16.5 & 57.8 \\
\hline $3^{\text {rd }}$ & $3.20 \pm 1.0$ & 22.1 & 64.8 & $4^{\text {th }}$ & $4.12 \pm 1.2$ & 16.9 & 59.6 \\
\hline $4^{\text {th }}$ & $8.97 \pm 0.8$ & 22.1 & 57.0 & Mean & \multicolumn{3}{|l|}{$3.58 \pm 0.26$} \\
\hline $5^{\text {th }}$ & $3.75+1.4$ & 21.4 & 73.3 & Jan.,20121 $1^{\text {st }}$ & $14.5+1.4$ & 17.6 & 51.0 \\
\hline Mean & \multicolumn{3}{|l|}{$4.22 \pm 1.21$} & $2^{\text {nd }}$ & $5.80 \pm 1.8$ & 16.6 & 60.8 \\
\hline Jan., $20111^{\text {st }}$ & $3.87 \pm 1.6$ & 22.2 & 68.6 & $3^{\text {rd }}$ & $8.40 \pm 2.2$ & 16.9 & 61.4 \\
\hline $2^{\text {nd }}$ & $4.14+1.3$ & 21.2 & 64.6 & $4^{\text {th }}$ & $9.02 \pm 1.7$ & 15.2 & 60.0 \\
\hline $3^{\text {rd }}$ & $4.58+2.1$ & 20.6 & 65.6 & $5^{\text {th }}$ & $12.07+2.3$ & 17.3 & 60.0 \\
\hline $4^{\text {th }}$ & $8.14+1.7$ & 20.1 & 63.3 & Mean & \multicolumn{3}{|l|}{$9.96+1.51$} \\
\hline Mean & \multicolumn{3}{|l|}{$5.18 \pm 1.00$} & Feb., $\quad 1^{\text {st }}$ & $17.0 \pm 4.5$ & 18.1 & 59.6 \\
\hline Feb., & $11.91+2.7$ & 19.5 & 63.5 & $2^{\text {nd }}$ & $24.2+5.1$ & 16.1 & 60.1 \\
\hline $2^{\text {nd }}$ & $24.90+3.0$ & 19.5 & 58.3 & $3^{\text {rd }}$ & $37.35+4.7$ & 17.3 & 60.1 \\
\hline $3^{\text {rd }}$ & $19.31+3.7$ & 25.6 & 60.8 & $4^{\text {th }}$ & $44.07 \pm 3.8$ & 19.4 & 60.7 \\
\hline $4^{\text {th }}$ & $15.30 \pm 3.2$ & 24.4 & 63.1 & Mean & \multicolumn{3}{|l|}{$30.65 \pm 6.14$} \\
\hline Mean & \multicolumn{3}{|l|}{$17.85 \pm 2.80$} & Mar., $1^{\text {st }}$ & $39.6 \pm 6.3$ & 19.9 & 60.6 \\
\hline March $1^{\text {st }}$ & $31.2+4.1$ & 21.6 & 65.8 & $2^{\text {nd }}$ & $41.2+4.9$ & 17.6 & 59.1 \\
\hline $2^{\text {nd }}$ & $28.7 \pm 3.3$ & 25.9 & 61.7 & $3^{\text {rd }}$ & $38.7 \pm 6.3$ & 18.0 & 58.4 \\
\hline $3^{\text {rd }}$ & $24.2+2.9$ & 26.7 & 65.6 & $4^{\text {th }}$ & $29.2 \pm 3.1$ & 21.1 & 59.0 \\
\hline $4^{\text {th }}$ & $16.3 \pm 3.5$ & 21.8 & 63.7 & Mean & $37.17 \pm 2.71$ & & \\
\hline $5^{\text {th }}$ & $14.51+2.9$ & 23.9 & 59.7 & Apr., $\quad 1^{\text {st }}$ & $17.2+4.5$ & 20.7 & 57.6 \\
\hline Mean & \multicolumn{3}{|l|}{$22.98+3.30$} & $2^{\text {nd }}$ & $19.1+2.9$ & 21.7 & 59.1 \\
\hline Apr., $\quad 1^{\text {st }}$ & $5.4 \pm 2.1$ & 27.3 & 57.0 & Mean & $18.15 \pm 0.95$ & & \\
\hline General mean & $9.56+3.54$ & & & General mean & $16.97+5.89$ & & \\
\hline
\end{tabular}


during the $3^{\text {rd }}$ week of December and February. On the other hand, Saleh et al., 1972 and Abdel-Samad, 1996 mentioned that the total number of $A$. craccivora on faba bean plants reached its maximum during the last week of March. In contrast, Selim et al., (1987) stated that $A$. craccivora had one peak on broad bean on $27^{\text {th }}$ of January 1980, then declined rapidly during March.

\subsection{On cowpea plants:}

The data in 2011 season, showed that the mean population density of $A$. craccivora on cowpea plants gradually increased as the growing season progressed to reach its maximum, (130.1 aphids/ plant) in the third week of July. After that, the mean number of aphids gradually decreased towards the end of the season Table, (2).

Table 2. Seasonal abundance of Aphis craccivora on cowpea plants, during 2011 and 2012 seasons, in the newly reclaimed sandy area, El-Khattara district.

\begin{tabular}{|c|c|c|c|c|c|c|}
\hline \multirow{3}{*}{$\begin{array}{l}\text { Date of inspection } \\
\text { (weeks) }\end{array}$} & \multicolumn{3}{|c|}{ The first season 2011} & \multicolumn{3}{|c|}{ The second season 2012} \\
\hline & \multicolumn{3}{|c|}{ Means } & \multicolumn{3}{|c|}{ Means } \\
\hline & Aphid /plant & $\begin{array}{l}\text { Temp. } \\
{ }^{\circ} \mathrm{C}\end{array}$ & $\begin{array}{c}\text { R.H. } \\
\%\end{array}$ & $\begin{array}{l}\text { Aphid } \\
\text { /plant }\end{array}$ & $\begin{array}{l}\text { Temp. } \\
{ }^{\circ} \mathrm{C}\end{array}$ & $\begin{array}{c}\text { R.H. } \\
\%\end{array}$ \\
\hline Jun. , & $4.3 \pm 1.3$ & 30.5 & 63.6 & $2.1 \pm 0.8$ & 26.5 & 58.4 \\
\hline $4^{\text {th }}$ & $15.9 \pm 2.4$ & 32.7 & 64.3 & $10.6 \pm 1.7$ & 27.8 & 59.0 \\
\hline Mean & $10.1 \pm 5.8$ & & & $6.35 \pm 4.3$ & & \\
\hline Jul., & $26.1 \pm 5.0$ & 34.0 & 67.4 & $16.34 \pm 2.9$ & 29.6 & 59.4 \\
\hline $2^{\text {nd }}$ & $49.6 \pm 4.7$ & 32.9 & 64.1 & $33.02 \pm 4.5$ & 30.7 & 64.0 \\
\hline $3^{\text {rd }}$ & $130.1 \pm 6.4$ & 34.2 & 70.0 & $76.4 \pm 6.1$ & 30.5 & 64.1 \\
\hline $4^{\text {th }}$ & $121.5 \pm 5.6$ & 34.8 & 70.4 & $99.81 \pm 5.3$ & 31.4 & 62.6 \\
\hline Mean & $81.83+25.9$ & & & $56.4 \pm 19.2$ & & \\
\hline Aug., & $101.4 \pm 3.8$ & 35.3 & 70.1 & $69.0 \pm 4.8$ & 31.3 & 59.7 \\
\hline $2^{\text {nd }}$ & $84.9 \pm 6.1$ & 35.4 & 73.3 & $41.2 \pm 3.5$ & 29.5 & 63.3 \\
\hline $3^{\text {rd }}$ & $31.7 \pm 3.4$ & 36.3 & 73.4 & $18.4 \pm 2.7$ & 27.5 & 63.4 \\
\hline $4^{\text {th }}$ & $20.5 \pm 2.7$ & 34.1 & 72.0 & $11.3 \pm 1.9$ & 29.8 & 68.3 \\
\hline Mean & $59.63 \pm 19.8$ & & & $34.98 \pm 13.0$ & & \\
\hline Sept., & $11.4 \pm 3.1$ & 31.4 & 67.0 & $7.8 \pm 1.1$ & 28.4 & 61.0 \\
\hline $2^{\text {nd }}$ & $5.6 \pm 4.3$ & 29.7 & 66.0 & $3.5 \pm 0.6$ & 28.5 & 61.6 \\
\hline $3^{\text {rd }}$ & $4.1 \pm 1.3$ & 28.2 & 64.3 & $2.3 \pm 0.9$ & 28.3 & 61.8 \\
\hline $4^{\text {th }}$ & $1.9 \pm 0.9$ & 28.3 & 62.6 & 0 & 28.4 & 62.0 \\
\hline Mean & $5.75 \pm 2.0$ & & & $3.4 \pm 1.6$ & & \\
\hline General mean & $39.33 \pm 18.71$ & & & $25.28+12.58$ & & \\
\hline
\end{tabular}


In 2012 season, the general mean population density of $A$. craccivora on cowpea plants generally increased during the growing season to reach its peak of 99.81 aphids /plant in the fourth week of July. The mean numbers of $A$. craccivora during the two seasons were 39.33 and 25.28 individuals/ plant, respectively (Table, 2). The obtained results was similar to those of Saleh et al., (1972) in Egypt who stated that $A$. craccivora was the most injurious pest attacking broad bean and cowpea in upper Egypt owing to the favourable weather conditions.

\section{Population density of predators attacking Aphis craccivora on faba bean and cowpea plants during the period of study:}

\subsection{On faba bean plants:}

Six species of predators were recorded during the two seasons of this study. These predators were Coccinella undecimpunctata L., Chrysoperla carnea (Steph.), Paederus alfierii (Koch), Syrphus sp., Orius sp. and Scymnus sp., Table, (3).

The occurrence of these predators in the two seasons could be descendingly arranged as follow: $C$. undecimpunctata was the dominant predator species with a rate of occurrence reached $31.26 \%$ and $29.16 \%$ from the total numbers of predators found during the two seasons, respectively. C. carnea represented the second category with a percent of $18.45 \%$ and $20.96 \%$ in the first and the second season, respectively. The rest predators arranged descendingly as follow, P. alfierii (17.33\%), Orius sp. (12.62\%), Syrphus sp. (11.3\%) and Scymnus sp. (9.04\%) in the first season Table, (3), whereas in the second season they arranged descendingly as follow, P. alfierii (15.03\%), Scymnus sp. (13.21\%), Orius sp. (11.39\%) and Syrphus sp. (10.25\%) Table, (4).

In the first season, the population density of whole predators started with low densities till reached its maximum (79 individuals/ 100 plants) on the second week of March, then gradually decreased till the end of the season. One additional peak 37 individuals / 100 plants was found on the first week of February Table , (3).

In the second season, the total number of predators was fluctuated and increased gradually to reach its maximum, (61 individuals/100 plants) on the third week of February. An additional peak was found in the second week of March, (58 individuals/ 100 plants), respectively Table (4). These results are in accordance with the findings of Kesten , 1975 and Abdel-Samad, 1996 who reported that Coccinellids, Syrphids and Chrysopa sp. were the most important predators attacking A. craccivora . 
Table 3. Seasonal abundance of the predatory insects associated with Aphis craccivora on faba bean plants during 2010-2011 season in the newly reclaimed sandy area, El-Khattara district.

\begin{tabular}{|c|c|c|c|c|c|c|c|c|c|}
\hline \multirow{2}{*}{$\begin{array}{c}\text { Date of } \\
\text { inspection } \\
\text { (weeks) }\end{array}$} & \multirow{2}{*}{ 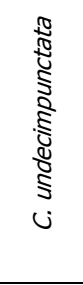 } & \multirow{2}{*}{$\begin{array}{l}\text { \& } \\
\frac{8}{0} \\
ن\end{array}$} & \multirow{2}{*}{$\begin{array}{l}: \\
\frac{\$}{2} \\
0\end{array}$} & \multirow{2}{*}{ 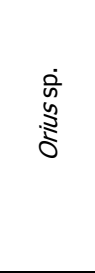 } & \multirow{2}{*}{ 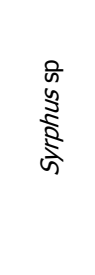 } & \multirow{2}{*}{ 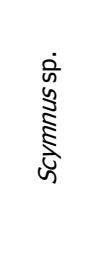 } & \multirow{2}{*}{ Total } & \multicolumn{2}{|c|}{$\begin{array}{c}\text { Corresponding means } \\
\text { of }\end{array}$} \\
\hline & & & & & & & & $\begin{array}{c}\text { Temp. } \\
{ }^{\circ} \mathrm{C}\end{array}$ & $\begin{array}{c}\text { R.H. } \\
\%\end{array}$ \\
\hline Nov., $3^{\text {rd }}$ & 0 & 0 & 0 & 0 & 0 & 0 & 0 & 24.9 & 65.3 \\
\hline $4^{\text {th }}$ & 2 & 0 & 0 & 0 & 0 & 0 & 2 & 23.7 & 65.4 \\
\hline Mean & $\begin{array}{c}1.0 \\
\pm 1.0 \\
\end{array}$ & 0 & 0 & 0 & 0 & 0 & $\begin{array}{c}1.0 \\
\pm 1.0 \\
\end{array}$ & & \\
\hline Dec., $1^{\text {st }}$ & 2 & 0 & 0 & 0 & 0 & 0 & 2 & 22.1 & 70.8 \\
\hline $2^{\text {nd }}$ & 3 & 0 & 1 & 0 & 0 & 0 & 4 & 21.7 & 61.0 \\
\hline $3^{\text {rd }}$ & 5 & 0 & 0 & 0 & 0 & 1 & 6 & 22.1 & 64.8 \\
\hline $4^{\text {th }}$ & 6 & 1 & 2 & 0 & 2 & 2 & 13 & 22.1 & 57.0 \\
\hline $5^{\text {th }}$ & 12 & 2 & 0 & 0 & 0 & 4 & 18 & 21.4 & 73.3 \\
\hline Mean & $\begin{array}{r}5.6 \\
+1.75 \\
\end{array}$ & $\begin{array}{c}0.6 \\
+0.4 \\
\end{array}$ & $\begin{array}{c}0.6 \\
+0.4 \\
\end{array}$ & 0 & $\begin{array}{c}0.4 \\
+0.39 \\
\end{array}$ & $\begin{array}{c}1.4 \\
+0.75 \\
\end{array}$ & $\begin{array}{c}8.6 \\
+2.9 \\
\end{array}$ & & \\
\hline $\begin{array}{r}\text { Jan., } 2011 \\
1^{\text {st }}\end{array}$ & 8 & 1 & 1 & 2 & 3 & 2 & 17 & 22.2 & 68.6 \\
\hline $2^{\text {nd }}$ & 6 & 3 & 3 & 1 & 2 & 4 & 19 & 21.2 & 64.6 \\
\hline $3^{\text {rd }}$ & 3 & 4 & 2 & 3 & 1 & 1 & 14 & 20.6 & 65.6 \\
\hline $4^{\text {th }}$ & 8 & 8 & 4 & 2 & 2 & 2 & 26 & 20.1 & 63.3 \\
\hline Mean & $\begin{array}{r}6.25 \\
+1.18 \\
\end{array}$ & $\begin{array}{r}4.0 \\
\pm 1.5 \\
\end{array}$ & $\begin{array}{r}2.5 \\
+0.64 \\
\end{array}$ & $\begin{array}{c}2.0 \\
\pm 0.4 \\
\end{array}$ & $\begin{array}{r}2.0 \\
\pm 0.41 \\
\end{array}$ & $\begin{array}{r}2.25 \\
\pm 0.63 \\
\end{array}$ & $\begin{array}{r}19.0 \\
\pm 2.5 \\
\end{array}$ & & \\
\hline Feb., $1^{\text {st }}$ & 14 & 6 & 8 & 2 & 4 & 3 & 37 & 19.5 & 63.5 \\
\hline $2^{\text {nd }}$ & 16 & 4 & 6 & 4 & 3 & 2 & 35 & 19.6 & 58.3 \\
\hline $3^{\text {rd }}$ & 15 & 14 & 9 & 6 & 4 & 4 & 52 & 25.6 & 60.8 \\
\hline $4^{\text {th }}$ & 14 & 11 & 6 & 9 & 6 & 7 & 53 & 24.4 & 63.1 \\
\hline Mean & $\begin{array}{r}14.75 \\
+0.48 \\
\end{array}$ & $\begin{array}{r}8.75 \\
+2.3 \\
\end{array}$ & $\begin{array}{r}7.25 \\
+0.75 \\
\end{array}$ & $\begin{array}{r}5.25 \\
+1.49 \\
\end{array}$ & $\begin{array}{r}4.25 \\
+0.63 \\
\end{array}$ & $\begin{array}{r}4.0 \\
+1.08 \\
\end{array}$ & $\begin{array}{r}44.25 \\
+4.78 \\
\end{array}$ & & \\
\hline March, $1^{\text {st }}$ & 16 & 13 & 13 & 11 & 7 & 5 & 65 & 21.6 & 65.8 \\
\hline $2^{\text {nd }}$ & 20 & 14 & 15 & 15 & 9 & 6 & 79 & 25.9 & 61.7 \\
\hline $3^{\text {rd }}$ & 12 & 8 & 11 & 6 & 3 & 2 & 42 & 26.7 & 65.6 \\
\hline $4^{\text {th }}$ & 2 & 5 & 8 & 4 & 8 & 3 & 30 & 21.8 & 63.7 \\
\hline $5^{\text {th }}$ & 0 & 3 & 2 & 2 & 4 & 0 & 11 & 23.9 & 59.7 \\
\hline Mean & $\begin{array}{r}10.0 \\
+3.9 \\
\end{array}$ & $\begin{array}{r}8.6 \\
+2.2 \\
\end{array}$ & $\begin{array}{r}9.8 \\
+2.26 \\
\end{array}$ & $\begin{array}{r}7.5 \\
+2.37 \\
\end{array}$ & $\begin{array}{r}6.9 \\
\pm 1.16 \\
\end{array}$ & $\begin{array}{r}3.2 \\
\pm 1.06 \\
\end{array}$ & $\begin{array}{r}45.4 \\
+13.56 \\
\end{array}$ & & \\
\hline Apri., $1^{\text {st }}$ & 2 & 1 & 1 & 0 & 2 & 0 & 6 & 27.3 & 57.0 \\
\hline Total & 166 & 98 & 92 & 67 & 60 & 48 & 531 & & \\
\hline$\%$ & 31.26 & 18.45 & 17.33 & 12.62 & 11.30 & 9.04 & 100 & & \\
\hline General mean & $\begin{array}{c}6.6 \pm \\
2.1\end{array}$ & $\begin{array}{c}3.83 \pm \\
1.6\end{array}$ & $\begin{array}{c}3.52 \pm \\
1.65\end{array}$ & $\begin{array}{c}2.46 \pm \\
1.31\end{array}$ & $\begin{array}{c}2.59 \pm \\
1.06\end{array}$ & $\begin{array}{c}1.81 \pm \\
0.67\end{array}$ & $\begin{array}{c}20.71 \pm \\
8.0\end{array}$ & & \\
\hline
\end{tabular}


Table 4. Seasonal abundance of the predatory insects associated with Aphis craccivora on faba bean plants during 2011-2012 season in the newly reclaimed sandy area, El-Khattara district.

\begin{tabular}{|c|c|c|c|c|c|c|c|c|c|}
\hline \multirow{2}{*}{$\begin{array}{l}\text { Date of } \\
\text { inspection } \\
\text { ( weeks) }\end{array}$} & \multirow{2}{*}{ 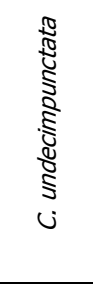 } & \multirow{2}{*}{ ن } & \multirow{2}{*}{$\begin{array}{l}: \\
\frac{\mathbb{2}}{\pi} \\
0\end{array}$} & \multirow{2}{*}{ 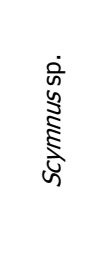 } & \multirow{2}{*}{ 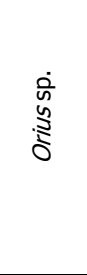 } & \multirow{2}{*}{$\begin{array}{l}00 \\
\text { जी } \\
\text { है } \\
\text { है } \\
\text { जी }\end{array}$} & \multirow{2}{*}{ Total } & \multicolumn{2}{|c|}{$\begin{array}{l}\text { Corresponding } \\
\text { means of }\end{array}$} \\
\hline & & & & & & & & $\begin{array}{c}\text { Temp. } \\
{ }^{\circ} \mathrm{C}\end{array}$ & $\begin{array}{c}\text { R.H. } \\
\%\end{array}$ \\
\hline Nov., $4^{\text {th }}$ & 0 & 0 & 0 & 0 & 0 & 0 & 0 & 21.8 & 61.2 \\
\hline Mean & 0 & 0 & 0 & 0 & 0 & 0 & 0 & & \\
\hline Dec., $1^{\text {st }}$ & 1 & 0 & 0 & 0 & 0 & 0 & 1 & 22.3 & 66.4 \\
\hline $2^{\text {nd }}$ & 3 & 0 & 0 & 0 & 0 & 1 & 4 & 19.8 & 61.6 \\
\hline $3^{\text {rd }}$ & 2 & 0 & 0 & 1 & 0 & 2 & 5 & 16.5 & 57.8 \\
\hline $4^{\text {th }}$ & 5 & 0 & 2 & 3 & 0 & 1 & 11 & 16.9 & 59.6 \\
\hline Mean & $\begin{array}{r}2.75 \\
\pm 0.9 \\
\end{array}$ & 0 & $\begin{array}{r}0.5 \\
+0.5 \\
\end{array}$ & $\begin{array}{c}1 \\
\pm 0.71 \\
\end{array}$ & 0 & $\begin{array}{c}1 \\
\pm 0.41 \\
\end{array}$ & $\begin{array}{r}5.25 \\
\pm 2.1 \\
\end{array}$ & & \\
\hline $\begin{array}{r}\text { Jan., } 2012 \\
1^{\text {st }} \\
\end{array}$ & 7 & 0 & 5 & 5 & 0 & 1 & 18 & 17.6 & 51.0 \\
\hline $2^{\text {nd }}$ & 5 & 1 & 3 & 2 & 0 & 2 & 13 & 16.6 & 60.8 \\
\hline $3^{\text {rd }}$ & 3 & 3 & 2 & 4 & 2 & 3 & 17 & 16.9 & 61.4 \\
\hline $4^{\text {th }}$ & 8 & 2 & 3 & 2 & 1 & 2 & 18 & 15.2 & 60.0 \\
\hline $5^{\text {th }}$ & 6 & 3 & 2 & 3 & 2 & 1 & 17 & 17.3 & 60.0 \\
\hline Mean & $\begin{array}{c}5.8 \\
+0.86 \\
\end{array}$ & $\begin{array}{c}1.8 \\
+0.58 \\
\end{array}$ & $\begin{array}{c}3.0 \\
+0.55 \\
\end{array}$ & $\begin{array}{r}3.2 \\
+0.65 \\
\end{array}$ & $\begin{array}{c}1 \\
+0.45 \\
\end{array}$ & $\begin{array}{r}1.8 \\
+0.37 \\
\end{array}$ & $\begin{array}{r}16.6 \\
+0.93 \\
\end{array}$ & & \\
\hline Feb., $1^{\text {st }}$ & 7 & 5 & 3 & 5 & 3 & 3 & 26 & 18.1 & 59.6 \\
\hline $2^{\text {nd }}$ & 8 & 10 & 4 & 3 & 2 & 3 & 30 & 16.1 & 60.1 \\
\hline $3^{\text {rd }}$ & 13 & 14 & 10 & 9 & 9 & 6 & 61 & 17.3 & 60.1 \\
\hline $4^{\text {th }}$ & 10 & 9 & 5 & 4 & 5 & 5 & 38 & 19.4 & 60.7 \\
\hline Mean & $\begin{array}{r}9.5 \\
\pm 1.32 \\
\end{array}$ & $\begin{array}{r}9.5 \\
\pm 1.8 \\
\end{array}$ & $\begin{array}{r}5.5 \\
\pm 1.6 \\
\end{array}$ & $\begin{array}{r}5.25 \\
\pm 1.3 \\
\end{array}$ & $\begin{array}{r}4.75 \\
\pm 1.5 \\
\end{array}$ & $\begin{array}{r}4.25 \\
\pm 0.8 \\
\end{array}$ & $\begin{array}{r}38.75 \\
\pm 7.82 \\
\end{array}$ & & \\
\hline March, $1^{\text {st }}$ & 11 & 13 & 6 & 3 & 5 & 4 & 42 & 19.9 & 60.6 \\
\hline $2^{\text {nd }}$ & 15 & 9 & 10 & 8 & 10 & 6 & 58 & 17.6 & 59.1 \\
\hline $3^{\text {rd }}$ & 9 & 10 & 5 & 3 & 7 & 3 & 37 & 18.0 & 58.4 \\
\hline $4^{\text {th }}$ & 5 & 7 & 3 & 2 & 3 & 1 & 21 & 21.1 & 59.0 \\
\hline Mean & $\begin{array}{r}10 \\
+2.1 \\
\end{array}$ & $\begin{array}{r}9.75 \\
\pm 1.25 \\
\end{array}$ & $\begin{array}{c}6.0 \\
+1.47 \\
\end{array}$ & $\begin{array}{c}4 \\
+1.35 \\
\end{array}$ & $\begin{array}{r}6.25 \\
\pm 1.5 \\
\end{array}$ & $\begin{array}{r}3.5 \\
+1.04 \\
\end{array}$ & $\begin{array}{r}37.75 \\
\pm 8.0 \\
\end{array}$ & & \\
\hline Apri., $1^{\text {st }}$ & 8 & 6 & 2 & 1 & 1 & 1 & 19 & 20.7 & 57.6 \\
\hline $2^{\text {nd }}$ & 2 & 0 & 1 & 0 & 0 & 0 & 3 & 21.7 & 59.1 \\
\hline Mean & $5 \pm 3.0$ & $\begin{array}{r}3 \\
+3.0 \\
\end{array}$ & $\begin{array}{r}1.5 \\
\pm 0.35 \\
\end{array}$ & $\begin{array}{r}0.5 \\
\pm 0.5 \\
\end{array}$ & $\begin{array}{r}0.5 \\
\pm 0.51 \\
\end{array}$ & $\begin{array}{c}0.5 \\
\pm 0.5 \\
\end{array}$ & $\begin{array}{r}10.5 \\
\pm 8.5 \\
\end{array}$ & & \\
\hline Total & 128 & 92 & 66 & 58 & 50 & 45 & 439 & & \\
\hline$\%$ & 29.16 & 20.96 & 15.03 & 13.21 & 11.39 & 10.25 & 100 & & \\
\hline General mean & $\begin{array}{c}5.51 \pm \\
1.57\end{array}$ & $\begin{array}{l}4.0 \pm \\
1.84\end{array}$ & $\begin{array}{c}2.75 \pm \\
1.04\end{array}$ & $\begin{array}{c}2.32 \pm \\
0.87\end{array}$ & $\begin{array}{c}2.08 \pm \\
1.11\end{array}$ & $\begin{array}{c}1.84 \pm \\
0.69\end{array}$ & $\begin{array}{c}18.14 \pm \\
6.75\end{array}$ & & \\
\hline
\end{tabular}




\subsection{On cowpea plants:}

Seven species of predators were found during the two seasons of this investigation. These predators were C. undecimpunctata, Syrphus sp., C. carnea, Scymnus sp., Orius sp., Cydonia vicina isis (Muls) and $P$. alfierii . The occurrence of these predators in the first season 2011 could be descendingly arranged as follow:

C. undecimpunctata was the dominant predator species with a rate of occurrence reached, $33.48 \%$. Syrphus sp. represented the second one with a percent of $18.06 \%$ from the total numbers of predators found in the first season, whereas the rest predator arranged descendingly as follow, C. carnea $13.22 \%$, Scymnus sp., $12.11 \%$, Orius sp., $11.23 \%$, C. Vicina isis $7.27 \%$ and $P$. alfierii $4.63 \%$ (Table 5).

In 2011 season, the population density of whole predators started with low densities till reached its maximum (89 individuals/ 50 plants) on the fourth week of July. One additional peak (69 individuals/ 50 plants) was found in the second week of August (Table, 5).

The occurrence of the same predators in the second season 2012 could be descendingly arranged as follow:

C. undecimpunctata was the dominant predator species with a rate of occurrence reached $(30.65 \%)$ from the total number of predators. Syrphus sp. (19.36\%), scymnus sp., (16.33\%), C. carnea (14.57\%), Orius sp., (8.79\%), C. vicina isis (5.78\%) and P. alfierii (4.25\%) (Table 6).

In 2012 season, the total number of predators was fluctuated and increased gradually to reach its maximum (78 individuals/ 50 plants) in the third week of July, (Table, 6).

The present results agree with those of Metwally (1998) who stated that, C. undecimpunctata, S. interuptus and $O$. albidipennis were associating with $A$. craccivora on cowpea plants. Abd-Elsamed et al., (2011) mentioned that $C$. undecimpunctata was represented by 16.88 and $23.96 \%, C$. vicina isis , 7.14 and $5.73 \%$, C. vicina nilotica 4.11 and $3.65 \%$, M. corollae 3.25 and $2.86 \%, C$. carnea 3.68 and $3.13 \%$ on $A$. craccivora infested cowpea during 2009 and 2010 seasons, respectively in Egypt. 
Table 5. Seasonal abundance of the predatory insects associated with Aphis craccivora on cowpea plants during 2011season in the newly reclaimed sandy area, ElKhattara district.

\begin{tabular}{|c|c|c|c|c|c|c|c|c|c|c|}
\hline \multirow{2}{*}{$\begin{array}{c}\text { Date of } \\
\text { inspection } \\
\text { ( weeks) }\end{array}$} & \multirow{2}{*}{ 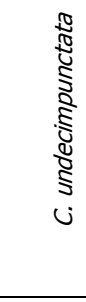 } & \multirow{2}{*}{$\begin{array}{l}\dot{0} \\
\text { n } \\
\text { के } \\
\frac{1}{2} \\
\text { जे }\end{array}$} & \multirow{2}{*}{ ن } & \multirow{2}{*}{ 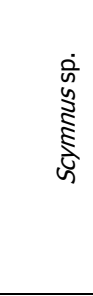 } & \multirow{2}{*}{ 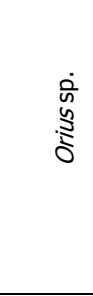 } & \multirow{2}{*}{ 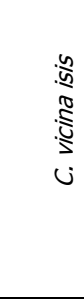 } & \multirow{2}{*}{ 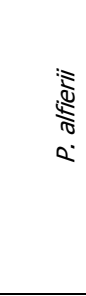 } & \multirow{2}{*}{ Total } & \multicolumn{2}{|c|}{$\begin{array}{c}\text { Corresponding } \\
\text { means of }\end{array}$} \\
\hline & & & & & & & & & $\begin{array}{l}\text { Temp. } \\
{ }^{\circ} \mathrm{C} \\
\end{array}$ & $\begin{array}{r}\text { R.H. } \\
\%\end{array}$ \\
\hline Jun., $3^{\text {rd }}$ & 0 & 0 & 0 & 0 & 0 & 0 & 0 & 0 & 26.5 & 58.4 \\
\hline $4^{\text {th }}$ & 3 & 3 & 2 & 1 & 0 & 1 & 0 & 10 & 27.8 & 59.0 \\
\hline Mean & $\begin{array}{r}1.5 \\
+1.5 \\
\end{array}$ & $\begin{array}{r}1.5 \\
+1.5 \\
\end{array}$ & $\begin{array}{l}1.0 \pm \\
1.0\end{array}$ & $\begin{array}{r}0.5 \\
+0.5 \\
\end{array}$ & 0 & $\begin{array}{r}0.5 \\
+0.5 \\
\end{array}$ & 0 & $\begin{array}{c}5.0 \pm \\
5\end{array}$ & & \\
\hline Jul., $\quad 1^{\text {st }}$ & 8 & 5 & 4 & 4 & 3 & 1 & 0 & 25 & 29.6 & 59.4 \\
\hline $2^{\text {nd }}$ & 17 & 15 & 6 & 9 & 7 & 2 & 2 & 58 & 30.7 & 64.0 \\
\hline $3^{\text {rd }}$ & 26 & 6 & 13 & 11 & 12 & 5 & 4 & 77 & 30.5 & 64.1 \\
\hline $4^{\text {th }}$ & 24 & 22 & 10 & 10 & 8 & 7 & 8 & 89 & 31.4 & 62.6 \\
\hline Mean & $\begin{array}{r}18.75 \\
+4.07 \\
\end{array}$ & $\begin{array}{r}12.0 \\
+4.02 \\
\end{array}$ & $\begin{array}{r}8.25 \\
+2.01 \\
\end{array}$ & $\begin{array}{r}8.5 \\
+1.6 \\
\end{array}$ & $\begin{array}{r}7.5 \\
+1.85 \\
\end{array}$ & $\begin{array}{r}3.75 \\
+1.38 \\
\end{array}$ & $\begin{array}{r}3.5 \\
+1.71 \\
\end{array}$ & $\begin{array}{r}62.25 \\
+13.96 \\
\end{array}$ & & \\
\hline Aug., $1^{\text {st }}$ & 16 & 7 & 7 & 5 & 6 & 3 & 2 & 46 & 31.3 & 59.7 \\
\hline $2^{\text {nd }}$ & 20 & 16 & 8 & 9 & 7 & 6 & 3 & 69 & 29.5 & 63.3 \\
\hline $3^{\text {rd }}$ & 14 & 3 & 5 & 4 & 4 & 4 & 2 & 36 & 27.5 & 63.4 \\
\hline $4^{\text {th }}$ & 12 & 2 & 3 & 2 & 1 & 3 & 0 & 23 & 29.8 & 68.3 \\
\hline Mean & $\begin{array}{r}15.5 \\
+1.7 \\
\end{array}$ & $\begin{array}{r}7.0 \\
+3.2 \\
\end{array}$ & $\begin{array}{r}5.75 \\
+1.11 \\
\end{array}$ & $\begin{array}{r}5.0 \\
+1.5 \\
\end{array}$ & $\begin{array}{r}4.5 \\
+1.3 \\
\end{array}$ & $\begin{array}{r}4.0 \\
+0.71 \\
\end{array}$ & $\begin{array}{r}1.75 \\
+0.63 \\
\end{array}$ & $\begin{array}{r}43.5 \\
+9.72 \\
\end{array}$ & & \\
\hline Sep., $1^{\text {st }}$ & 8 & 2 & 2 & 0 & 2 & 1 & 0 & 15 & 28.4 & 61.0 \\
\hline $2^{\text {nd }}$ & 4 & 1 & 0 & 0 & 1 & 0 & 0 & 6 & 28.5 & 61.6 \\
\hline $3^{\text {rd }}$ & 0 & 0 & 0 & 0 & 0 & 0 & 0 & 0 & 28.3 & 61.8 \\
\hline $4^{\text {th }}$ & 0 & 0 & 0 & 0 & 0 & 0 & 0 & 0 & 28.4 & 62.0 \\
\hline Mean & $\begin{array}{l}3.0 \pm \\
1.9 \\
\end{array}$ & $\begin{array}{r}0.75 \\
+0.48 \\
\end{array}$ & $\begin{array}{r}0.5 \\
+0.5 \\
\end{array}$ & 0 & $\begin{array}{r}0.75 \\
+0.48 \\
\end{array}$ & $\begin{array}{r}0.25 \\
+0.25 \\
\end{array}$ & 0 & $\begin{array}{r}8.5 \\
+6.65 \\
\end{array}$ & & \\
\hline Total & 152 & 82 & 60 & 55 & 51 & 33 & 21 & 454 & & \\
\hline$\%$ & 33.48 & 18.06 & 13.22 & 12.11 & 11.23 & 7.27 & 4.63 & 100 & & \\
\hline General mean & $\begin{array}{r}9.69 \\
+4.35 \\
\end{array}$ & $\begin{array}{r}5.31 \\
+2.63 \\
\end{array}$ & $\begin{array}{r}3.87 \\
+1.88 \\
\end{array}$ & $\begin{array}{r}3.5 \\
+2.0 \\
\end{array}$ & $\begin{array}{r}3.19 \\
+1.74 \\
\end{array}$ & $\begin{array}{r}2.12 \\
+1.01 \\
\end{array}$ & $\begin{array}{r}1.31 \\
+0.84 \\
\end{array}$ & $\begin{array}{r}30.5 \\
+14.11 \\
\end{array}$ & & \\
\hline
\end{tabular}


Table 6. Seasonal abundance of the predatory insects associated with Aphis craccivora on cowpea plants during 2012 season in the newly reclaimed sandy area, ElKhattara district.

\begin{tabular}{|c|c|c|c|c|c|c|c|c|c|c|}
\hline \multirow{2}{*}{$\begin{array}{c}\text { Date of } \\
\text { inspection } \\
\text { ( weeks) }\end{array}$} & \multirow{2}{*}{ 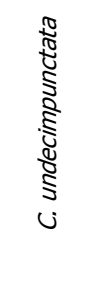 } & \multirow{2}{*}{$\begin{array}{l}\text { जी } \\
\text { है } \\
\text { है } \\
\text { जे }\end{array}$} & \multirow{2}{*}{$\begin{array}{l}\dot{0} \\
\text { जे } \\
\text { है } \\
\text { जे }\end{array}$} & \multirow{2}{*}{ ن } & \multirow{2}{*}{ 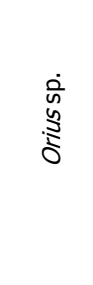 } & \multirow{2}{*}{ 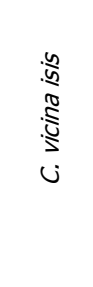 } & \multirow{2}{*}{$\begin{array}{l}: \\
\frac{\pi}{2} \\
0\end{array}$} & \multirow{2}{*}{ Total } & \multicolumn{2}{|c|}{$\begin{array}{c}\text { Corresponding } \\
\text { means of }\end{array}$} \\
\hline & & & & & & & & & $\begin{array}{c}\text { Temp. } \\
{ }^{\circ} \mathrm{C}\end{array}$ & $\begin{array}{c}\text { R.H. } \\
\%\end{array}$ \\
\hline Jun., $3^{\text {rd }}$ & 2 & 0 & 0 & 0 & 0 & 0 & 0 & 2 & 26.5 & 58.4 \\
\hline $4^{\text {th }}$ & 4 & 3 & 2 & 3 & 0 & 0 & 0 & 12 & 27.8 & 59.0 \\
\hline Mean & $\begin{array}{r}3.0 \\
\pm 1.0\end{array}$ & $\begin{array}{r}1.5 \\
\pm 1.5\end{array}$ & $\begin{array}{r}1.0 \\
\pm 1.0\end{array}$ & $\begin{array}{r}1.5 \\
\pm 1.5\end{array}$ & 0 & 0 & 0 & $\begin{array}{l}7 \pm \\
5.0\end{array}$ & & \\
\hline Jul., $\quad 1^{\text {st }}$ & 6 & 5 & 4 & 5 & 1 & 0 & 0 & 21 & 29.6 & 59.4 \\
\hline $2^{\text {nd }}$ & 10 & 7 & 6 & 6 & 4 & 1 & 3 & 37 & 30.7 & 64.0 \\
\hline $3^{\text {rd }}$ & 25 & 17 & 9 & 14 & 5 & 3 & 5 & 78 & 30.5 & 64.1 \\
\hline $4^{\text {th }}$ & 23 & 14 & 10 & 8 & 7 & 2 & 6 & 70 & 31.4 & 62.6 \\
\hline Mean & $\begin{array}{r}16.0 \\
\pm 4.71\end{array}$ & $\begin{array}{r}10.75 \\
\pm 2.84\end{array}$ & $\begin{array}{r}7.25 \\
\pm 1.38 \\
\end{array}$ & $\begin{array}{r}8.25 \\
\pm 2.02\end{array}$ & $\begin{array}{r}4.25 \\
\pm 1.25\end{array}$ & $\begin{array}{r}1.5 \\
\pm 0.65\end{array}$ & $\begin{array}{r}3.5 \\
\pm 1.32 \\
\end{array}$ & $\begin{array}{r}51.5 \\
\pm 13.49\end{array}$ & & \\
\hline Aug., $1^{\text {st }}$ & 17 & 10 & 13 & 7 & 7 & 5 & 2 & 61 & 31.3 & 59.7 \\
\hline $2^{\text {nd }}$ & 14 & 8 & 10 & 5 & 5 & 2 & 2 & 46 & 29.5 & 63.3 \\
\hline $3^{\text {rd }}$ & 8 & 6 & 7 & 3 & 3 & 4 & 0 & 31 & 27.5 & 63.4 \\
\hline $4^{\text {th }}$ & 6 & 5 & 4 & 4 & 1 & 2 & 0 & 22 & 29.8 & 63.3 \\
\hline Mean & $\begin{array}{r}11.25 \\
\pm 2.56\end{array}$ & $\begin{array}{r}7.25 \\
\pm 1.11\end{array}$ & $\begin{array}{r}8.5 \\
\pm 1.94 \\
\end{array}$ & $\begin{array}{r}4.75 \\
\pm 0.85\end{array}$ & $\begin{array}{r}4.0 \\
\pm 1.3\end{array}$ & $\begin{array}{r}3.25 \\
\pm 0.75\end{array}$ & $\begin{array}{r}1.0 \\
\pm 0.58 \\
\end{array}$ & $\begin{array}{r}40.0 \\
\pm 8.57\end{array}$ & & \\
\hline Sep., $1^{\text {st }}$ & 4 & 0 & 0 & 3 & 2 & 1 & 0 & 10 & 28.4 & 61.0 \\
\hline $2^{\text {nd }}$ & 3 & 2 & 0 & 0 & 0 & 3 & 0 & 8 & 28.5 & 61.6 \\
\hline $3^{\text {rd }}$ & 0 & 0 & 0 & 0 & 0 & 0 & 0 & 0 & 28.3 & 61.8 \\
\hline $4^{\text {th }}$ & 0 & 0 & 0 & 0 & 0 & 0 & 0 & 0 & 28.4 & 62.0 \\
\hline Mean & $\begin{array}{r}1.75 \\
\pm 1.03\end{array}$ & $\begin{array}{r}0.5 \\
\pm 0.5\end{array}$ & 0 & $\begin{array}{r}0.75 \\
\pm 0.75\end{array}$ & $\begin{array}{r}0.5 \\
\pm 0.5\end{array}$ & $\begin{array}{c}1.0 \\
\pm 0.71\end{array}$ & 0 & $\begin{aligned} & 4.5 \\
+ & 2.63\end{aligned}$ & & \\
\hline Total & 122 & 77 & 65 & 58 & 35 & 23 & 18 & 398 & & \\
\hline$\%$ & 30.65 & 19.36 & 16.33 & 14.57 & 8.79 & 5.78 & 4.25 & 100 & & \\
\hline General mean & $\begin{array}{r}8.0 \\
+3.4\end{array}$ & $\begin{array}{r}5.0 \\
\pm 2.42\end{array}$ & $\begin{array}{r}4.19 \\
+2.15\end{array}$ & $\begin{array}{r}3.81 \\
\pm 1.72\end{array}$ & $\begin{array}{r}2.19 \\
\pm 1.12\end{array}$ & $\begin{array}{r}1.44 \\
\pm 0.68\end{array}$ & $\begin{array}{r}1.13 \\
\pm 0.83\end{array}$ & $\begin{array}{r}25.75 \\
\pm 11.79\end{array}$ & & \\
\hline
\end{tabular}




\section{Effect of temperature and relative humidity on the population densities of Aphis craccivora and its associated predators on faba bean and cowpea plants during two successive seasons:}

\subsection{On faba bean}

The correlation between number of $C$. undecimpunctata and mean relative humidity was negative highly significant $(r=0.6708)$. On the other hand, the correlation between the number of Scymnus sp. and maximum temperature was negative significantly correlation in the second season (Table , 7).

\subsection{On cowpea}

The results in Table, (7) revealed that highly positive significant correlation was found between number of $A$. craccivora and maximum temperature and mean relative humidity in the first season ( $r=0.6815$ and 0.7283 respectively), as well as, between the minimum temperature and $A$. craccivora in the second season $(r=0.7704)$. The correlation between the number of $C$. undecimpunctata $(r=0.5508)$ and $P$. alfierii $(r=0.6178)$ and maximum temperature was positive significantly correlation in the first season, while the number of $C$. carnea was positively correlated with the maximum temperature in both seasons, ( $r-0.6611$ and 0.5789 respectively). Meanwhile the correlation between the number of Orius sp., Scymnus sp. and the maximum temperature were highly positive significant correlation in the first season $(0.6663$ and 0.6852 respectively).

In the second season, also the correlation coefficient between the number of C. undecimpunctata and C. carnea was highly positive significant correlation with the minimum temperature ( $r=0.7231$ and 0.6954 , respectively) whereas the same results were obtained with the mean relative humidity, in the first season $(r=0.7557$ and 0.7262 ) respectively.

The correlation between the number of $P$. alfierii and Syrphus sp. with minimum temperature was highly positive significant in the second season, while it was positive significant with Scymnus sp. in the same season.

The correlation between the number of $P$. alfierii and Orius $\mathrm{sp}$. with the mean relative humidity, was positive significant ( 0.5999 and 0.6970 respectively) in the first season, meanwhile it was highly positive significant correlation with Scymnus sp. and C. vicina isis in the first season, respectively. These results are in general agree with those of Hassanein et al., (1992) and El-Maghraby et al., (2008) 
Table 7. Simple correlation between Aphis craccivora Koch. infested faba bean and cowpea plants and its associated predatory insects $\left(X_{1}\right)$ and maximum temperature $\left(X_{2}\right)$, minimum temperature $\left(X_{3}\right)$ and mean relative humidity $\left(X_{4}\right)$ during the two successive seasons in the newly reclaimed sandy area of El-Khattara district, Sharkia Governorate, Egypt

\begin{tabular}{|c|c|c|c|c|c|c|c|c|c|c|c|c|}
\hline \multirow{3}{*}{ Variable } & \multicolumn{6}{|c|}{ Faba bean } & \multicolumn{6}{|c|}{ Cowpea } \\
\hline & \multicolumn{2}{|c|}{$r 1-2$} & \multicolumn{2}{|c|}{$r 1-3$} & \multicolumn{2}{|c|}{$\mathrm{r} 1-4$} & \multicolumn{2}{|c|}{$\mathrm{r} 1-2$} & \multicolumn{2}{|c|}{$\mathrm{r} 1-3$} & \multicolumn{2}{|c|}{$\mathrm{r} 1-4$} \\
\hline & $2010 / 11$ & $2011 / 12$ & $2010 / 11$ & $2011 / 12$ & $2010 / 11$ & $2011 / 12$ & 2011 & 2012 & 2011 & 2012 & 2011 & 2012 \\
\hline 1.Aphid & & & & & & & & & & & & \\
\hline Aphis craccivora & 0.2398 & -0.3528 & 0.0389 & -0.1049 & 0.1783 & -0.0588 & $0.6815^{* *}$ & 0.4036 & -0.2052 & $0.7704 * *$ & $0.7238^{* *}$ & -0.0187 \\
\hline 2. Predatory insects & & & & & & & & & & & & \\
\hline C. undecimpunctata & 0.0843 & -03681 & -0.1910 & -0.0119 & -0.0142 & $-0.6708 * *$ & $0.5508^{*}$ & 0.4119 & -0.3121 & $0.7231^{* *}$ & $0.7557^{* *}$ & 0.0618 \\
\hline C. carnea & 0.2994 & -0.1959 & 0.0393 & -0.2682 & 0.2179 & -0.0097 & $0.6611^{*}$ & $0.5789 *$ & 0.2593 & $0.6954 * *$ & $0.7262 * *$ & 0.1013 \\
\hline P. alfierii & 0.2289 & -0.3529 & 0.0861 & 0.0924 & 0.1878 & -0.1335 & $0.6178^{*}$ & 0.4314 & -0.1526 & $0.7887^{* *}$ & 0.5999* & -0.0950 \\
\hline Orius sp. & 0.3285 & -0.2262 & 0.1473 & -0.2226 & 0.2286 & 0.0542 & $0.6663^{* *}$ & 0.2648 & -0.2392 & 0.6246 & $0.6970 *$ & 0.2069 \\
\hline Syrphus sp. & 0.2218 & -0.3794 & 0.0549 & -0.1974 & 0.1722 & 0.0244 & 0.3357 & 0.5002 & -0.0822 & $0.7564 * *$ & 0.2230 & 0.1314 \\
\hline Scymnus sp. & 0.1012 & $-0.5599 *$ & -0.1393 & 0.1464 & 0.2286 & -0.0729 & $0.6852 * *$ & 0.2958 & -0.2273 & $0.5436 *$ & $0.6988^{* *}$ & 0.3104 \\
\hline C. vicina isis & & & & & & & $0.5436 *$ & -0.0140 & -0.2461 & 0.3396 & $0.7643 * *$ & 0.4303 \\
\hline Total insect predators & 0.2329 & -0.3528 & -0.0054 & -0.0995 & 0.1563 & -0.0378 & $0.6582 *$ & 0.4237 & -0.2642 & $0.7172 * *$ & $0.7399 * *$ & 0.1542 \\
\hline
\end{tabular}




\section{REFERENCES}

1. Abdel-Rahman , G.A., M.H. Belal, N.M. Ibrahim and E.A. Ali, 2005. Observations on toxic effects of some desert plant extracts on the cowpea aphid, Aphis craccivora Koch. Egypt J. Agric. Res., 83 (2): 609-621.

2. Abdel-Samad, S.S. M. 1996. Studies on natural enemies on certain insects attacking leguminous crop. M.Sc. Thesis, Fac. of Agric. Ain Shams University, 94pp.

3. Abd-Elsamed, A.A., M.S. Hashem and A.A.A. Saleh. 2011. Effect of some intercropping systems and potassium fertilization rate for maize with cowpea crops on the population density of certain pests and their natural enemies in Sharkia Governorate, Egypt. J. Plant Prot. And Path. Mansoura Univ., 2 (2): 153-167.

4. El-Defrawi, G.M., K. Azza Emam, I.A. Marzouk and L. Rizkalla, 2000. Population dynamics and seasonal distribution of Aphis craccivora Koch. and associated natural enemies in relation to virus disease incidence in faba bean fields. Egypt . J. Agric. Res., 78 (2): 627-641.

5. El-Heneidy, A., G. Resk, A.M. Hekal and S. Abdel-Samad. 1998. Impact of planting date of aphid populations and associated natural enemies on faba bean plants in Egypt. Arab. J. Plant Protection, 16 (2): 55-59.

6. El-Maghraby, M.M.A., M.M. El-Zohairy, Aziza, M. El-Gantiry and Sh. A.M.Ali. 2008. Relationship between certain aphid species infesting leaves of mandarin trees and navel orange and associated aphidophagous insects in El-Khattara district, Sharkia Governorate, Egypt. J. Appl. Sci., 23 (10A): 352-373.

7. Hassanein, S.S.M., S.S.A.El-Shakaa and M.M.A. El-Maghraby. 1992. Seasonal occurrence of some leaf insects attacking forage cowpea and guar plants at Zagazig region. J. Agric. Sci. Mansoura Univ., 18 (4): 1221-1234.

8. Ibrahim, A.M.A. 1994. Aphids and their parasitoids on apple trees at Giza Region. Egypt, J. Biol. Pest Control 4 (1): 35-43.

9. Kesten, L.A. 1975. Insect enemies of the Lucerne aphid. Zashchita Rastenii (11): 28.

10. Kuroli, G., K. Pocsai and L. Nemeth. 1999. Flight activity and population changes of aphids in faba bean. Novenyvedelem, 35 (11): 545-554.

11. Maghraby, H.M.M. 2012. Studies on the parasitoid Diaeretiella rapae on some aphid species in Sharkia Governorate. M.Sc. Thesis, Fac. of Agric., Moshtohor, Benha Univ. pp. 222. 
12. Mandour, N.S., N.A.S.El-Basha and T. X. Liu. 2006. Functional response of the ladybird Cydonia vicina nilotica to cowpea aphid, Aphis craccivora in the laboratory. Insect Sci., 13 (1): 49-54.

13. Metwally, S.A.G. 1998. Predators and their role in controlling aphid population. Egyptian . J. Agric. Res., 76 (1): 105-116.

14. Ortiz, V., E. Navarro, S., Castro, G. Carazo and J. Romero. 2006. Incidence and transmission of faba beans necrotic yellows virus (FB NYV) in Spain. Spanish. J Agric. Res., 4 (3): 255-260.

15. Saleh, M.R.A., M. H. Hassanein and A.H. El-Sebae. 1972. Population dynamica of Aphis craccivora Koch. on broad bean and cowpea in upper Egypt (Homoptera: Aphididae). Bull. Soc. Ent. Egypt (56): 135-138.

16. SAS Institute Inc. 1999. SAS/STAT user's guide. 6-12 edition Cary, NC, SAS. Institute Inc.

17. Selim, A.A., S.A.El-Refai and A. El-Gantiry. 1987. Seasonal fluctuations in the population of Aphid cracciovra Koch., Myzus persicae (Sulz.) Aphis gossypii (Glov.) and their parasites. Ann. Agric. Sci. Ain Shams Univ., 32 (3): 1837-1848.

18. Sharma, R. P. and Yadav. 1994. Population dynamics of bean aphid (Aphis craccivora Koch.) and its predatory complex in relation to crop type (lentil, Lathyrus and faba bean) and weather conditions. J. Ent. Res., 18 (1): 25-36.

19. Srikanth, J. and N.H. Lakkundi. 1990. Seasonal population fluctuations of the cowpea aphid, Aphis craccivora Koch. and its predatory, Coccinellids. Insect Sci. and its App.,11 (1): 21-26. 


\section{من اللوبيا Aphis craccivora Koch ومفترساته الحشرية على نباتات الفول البلدى واللوبيا فى المناطق الرملية المستصلحة حديثا فى مصر}

شحتة على محمد على' ، أحمد أمين أحمد صالح' ، نادية الحسينى محمد r

1 - قسم بحوث الحشرات الثاقبة الماصنة ، معهد بحوث وقاية النباتات ، مركز البحوث الزراعيـة-

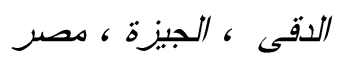

r- قسم بحوث المكافحة البيولوجية ، معهة بحوث وقاية النباتات - مركز البحوث النزراعبــة -

$$
\text { الدقى - الجبزة - مصر }
$$

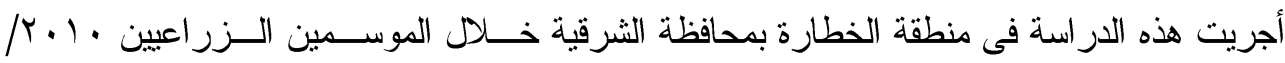

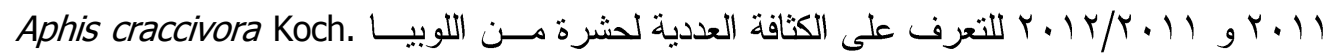
و المفترسات المصاحبة لها فى حقول الفول البلدى و اللوبيا.

أوضحت النتائج أن لمن اللوبيا ثلاث ذروات على محصول الفول البلدى خلال موسمى الدراســة

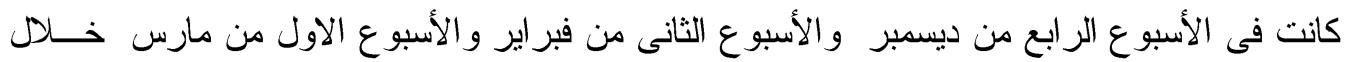

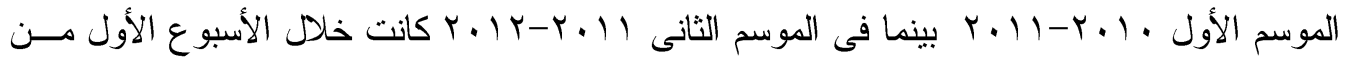
يناير و الأسبوع الرابع من فبر اير والأسبوع الثانى من مارس على محصول الثى الفول البلدى.

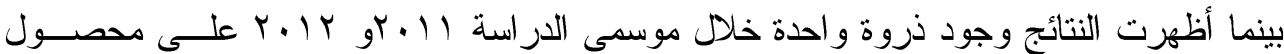

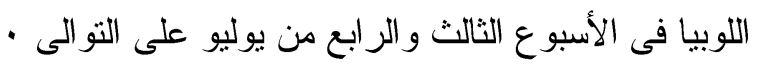

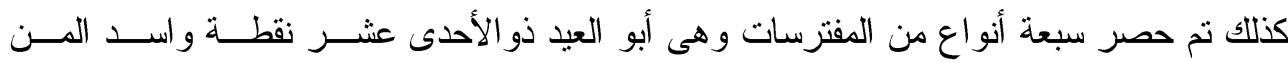

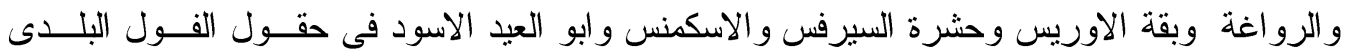
و اللوبيا و التى تؤثر بشكل معنوى على تطور وسير الإصابة بالمن.

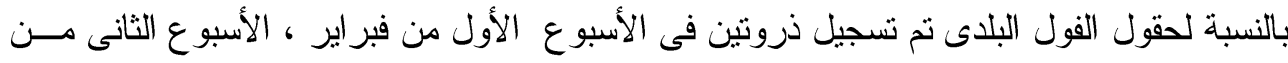

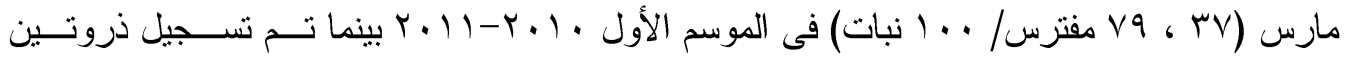

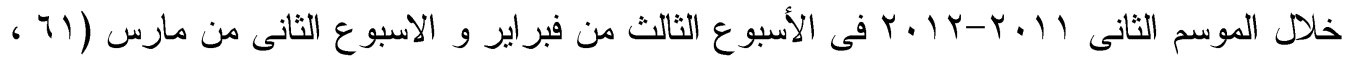

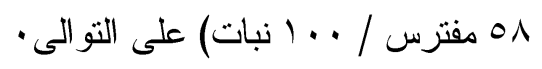

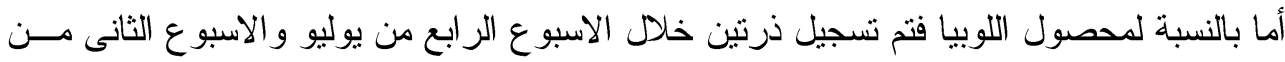

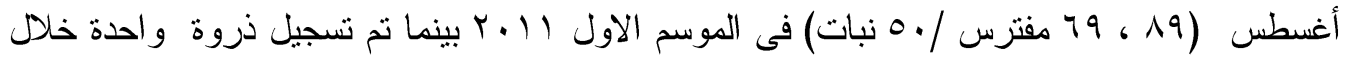

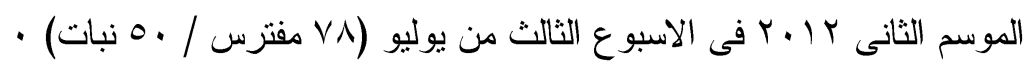

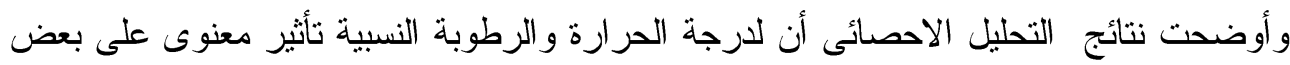

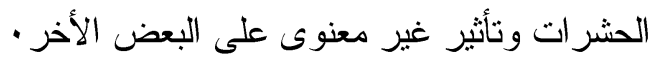

